\title{
Editorial
}

\section{Patient safety initiatives: are we ready for it?}

\author{
B Bhattarai, Head of Department, Department of Anaesthesiology and Critical Care,
} B.P.Koirala Institute of Health Sciences, Dharan

Patient safety is an issue of concern in healthcare both for the care providers and the consumers. Understandably, it is not possible to stop sentinel events from happening in healthcare; the bottom line is no one wants patients getting hurt. Therefore, prevention of errors and adverse effects associated with healthcare has remained the main aim of any patient safety initiative and patient safety has already been a discipline in itself.

Agency for Healthcare Research and Quality defines patient safety as a discipline in the health care sector that applies safety science methods toward the goal of achieving a trustworthy system of health care delivery ${ }^{1}$. Patient safety is also an attribute of health care system; it minimizes the incidence and impact of, and maximizes recovery from, adverse events.

Addition of new technologies, drugs and modalities in patient treatment is making our health care more and more complex day by day. The situation is becoming even more challenging as the elderly population with significant comorbidities is ever increasing due to improving economic conditions. In order to address the demand thus created, patient safety is evolving as an important subject focusing on reporting, analyzing, preventing and minimizing medical errors that can lead to adverse healthcare events.

According to WHO, one in every 10 patients becomes victim of medical error or unsafe care around the world in well funded and technologically advanced hospital setting 2 . Therefore, patient safety has been considered an endemic concern. Moreover, the situation is much less known from less advanced centres and non-hospital setting which actually contribute as the major health care providing outlets globally.
A healthcare error can simply be defined as a preventable adverse effect of care. Errors in health care can be attributed to several human related, system related and medical condition related factors such as drug treatment, injuries due to medical device, surgical and anaesthesia related errors, health care associated infections, unsafe injection practices, use of unsafe blood products, injury due to fall in hospitals, organizational determinants and latent failures, safety culture training and education of human resources stress fatigue, misdiagnosis, counterfeit and substandard drugs, poor test follow up, inadequate measure of patient safety, lack of patient involvement in patient safety etc $^{3}$.

Communication is one of the most important factors that can minimize adverse events in providing health care. Inadequate communication between health care providers, or between providers and the patient and or family/relatives, has been found to be the main root cause of more than half of the serious adverse events ${ }^{4}$. Inadequate patient assessment on part of the care providers and poor training are other important causes of adverse events. But it should not be misunderstood that incompetent care providers are the only common causes for mishaps. Many of the mishaps are actually the results of normal human lapses and not due to negligence ${ }^{5}$.

Although some errors such as surgery related ones may be more easily detected, they are occurring in all areas, all levels and all types of settings however advanced they may be. It should not be simply interpreted that high risk care means inevitable adverse events. Adverse outcome is more likely due to the severity of the disease itself than the complex procedure 
being undertaken ${ }^{6}$. Therefore, an adverse event should not always be equated with an error on the provider's part. In fact, many unforeseen complications and side effects occur from the disease condition or treatment itself.

Much is required to be done in order to improve patient safety and inculcate safety culture in a setting of resource constrained developing country like Nepal. It may be challenging but we do not need to wait for any appropriate time to take initiatives for such an issue as patient safety; any time is appropriate for it. Joint Commission, the organization that certifies and gives accreditation to healthcare organizations and programmes in the US has brought out some simple achievable patient safety goals for hospitals ${ }^{7}$. Ensuring patient identity, improving staff communications, safe use of drugs and medications, safe use of device alarms, prevention of infection, identification of safety risk in patient and prevention of surgical mistakes are the focus areas identified in the goals. If we pay proper attention to these areas we can certainly reduce the incidences of adverse events and improve patient safety in any type or level of healthcare setting.

Service providers in a resource limited setting like ours are required to be even more updated and prepared in patient safety issues. Unfortunately, the importance of awareness about patient safety related issues and prevention of adverse outcome is yet to be properly realized in such setting. Present is the best time for us to take initiatives in patient safety issues in whatever capacity we may be working.

\section{References}

1. Emanuel L. What exactly is patient safety? Agency for Healthcare Research and Quality. www.ahrq.com/downloads/pub/advances2/ vol1/advance/emanuel-berwick-110.pdf accessed on 10/01/2014

2. World Alliance for Patient Safety. World Health Organization. www.who. int/patientsafety/information centre/ documents/ps research-brochure en.pdf accessed on 10/01/2014

3. World Alliance for Patient Safety. Summary of the evidence on patient safety: Implications for research. http://whqlibdoc.who.int/ publications/2008/9789241596541 eng.pdf accessed on 10/01/2014

3. The Joint Commission's Annual Report on Quality and Safety 2007. Improving America's Hospitals. www.jointcommission. org/assets/1/6/2007_Annual_Report.pdf accessed on 10/01/2014

4. Weingart SN, Wilson RM, Gibberd RW, Harrison B. Epidemiology of medical errors. BMJ 2000; 320:774-7

5. Neale G, Woloshynowych M, Vincent C. Exploring the causes of adverse events in NHS hospital practice. J R Soc Med 2001; 94: $322-30$

6. The Joint Commission. Hospital: 2014 National Patient Safety Goals. www. jointcommission.org/hap_2014_npsgs/ accessed on 10/01/2014 